\title{
RASGOS ESQUELETALES DE ADAPTACIÓN AL CLIMA Y A LA ACTIVIDAD ENTRE LOS HABITANTES ABORÍGENES DE TIERRA DEL FUEGO
}

\author{
OSBJORN M. PEARSON* Y MARIO MILLONES*
}

\begin{abstract}
RESUMEN
Al momento de contacto con los europeos, existían dos grupos humanos que ocupaban Tierra del Fuego, presentando diferentes estrategias de subsistencia: los yámana a lo largo de las costas oeste y sur de la isla, y los selk'nam en el interior. Estas poblaciones, que soportaron uno de los climas más fríos de Sudamérica, representan una valiosa oportunidad para estudiar tanto sus adaptaciones a largo plazo al clima, como aquéllas a corto plazo relativas a sus actividades habituales. Estos distintos patrones de subsistencia permiten predecir diferentes robusteces y morfología de las diáfisis en los huesos largos de extremidades superiores e inferiores. Debido al clima, los niveles de robustez de extremidades y articulaciones, así como sus proporciones corporales, deberían ser similares a aquellos grupos humanos adaptados al frío.

Existen escasos restos esqueletales de miembros de estos grupos en Norte América y Europa. La muestra analizada corresponde a 14 esqueletos selk'nam y 6 yámana, pertenecientes a museos de Argentina y Chile, la que fue incrementada con datos relevados de publicaciones. Se calcularon y compararon los índices de robustez de las diáfisis y epífisis de los huesos largos, el ancho relativo bi-iliaco y la proporción AP/ML medial de las diáfisis. Los resultados obtenidos muestran que los fueguinos presentan huesos largos extremadamente robustos y pelvis relativamente anchas, similares a la mayor parte de las poblaciones recientes adaptadas al frío. Sin embargo, cuando los conjuntos se estratificaron por sexo, no se encontraron diferencias significativas entre las extremidades superiores e inferiores, ni tampoco entre los índices pilástrico y platicnémico. Debe por tanto tenerse cuidado en la interpretación de dichos rasgos, toda vez que la literatura etnográfica señala diferencias entre las actividades de ambos grupos, pero sus esqueletos no las manifiestan.
\end{abstract}

\section{SKELETAL FEATURES SHOWING ADAPTATION TO CLIMATE AND ACTIVITY OF ABORIGINAL INHABITANTS OF TIERRA DEL FUEGO}

\begin{abstract}
The people who lived on Tierra del Fuego Island at the time of European contact offer a remarkable opportunity to study the effects on the skeleton of long-term adaptation to climate and the short-term adaptation to habitual activity. The island provided one of the coldest habitats in South America. Two groups with different subsistence strategies occupied the island at contact: the Yámana along the southern and western coast and the Selk'nam in the
\end{abstract}

* University of New Mexico, Albuquerque, NM 87131-1086, Estados Unidos. Tel: (505) 277-6692, Fax: (505) 277-0874.

Correo electrónico (e-mail): ompear@unm.edu; millones@unm.edu 
interior. The dissimilar subsistence patterns would support contrasting predictions about robusticity and diaphyseal shapes of long bones in the upper and lower limb. Due to climate, both groups should show levels of limb and joint robusticity and body proportions similar to other cold adapted humans.

Skeletons of either group are rare in North America and Europe. Selk'nam and Yámana skeletons were measured at Museums in Argentina and Chile and supplemented with published data. Robusticity indices of the shafts and epiphyses of limb bones, relative bi-iliac breadth, and AP/ML midshaft ratios were calculated and compared. The results show the Fueguians had very robust limb bones and relatively broad pelves in common with the most cold-adapted recent populations. However, no significant differences existed in matched-sex comparisons of upper limb versus lower limb robusticity or in pilastric and platycnemic indices. The difference in ethnographically documented activity and lack of its skeletal manifestation argues for the use of caution in interpreting these traits.

\section{INTRODUCCIÓN}

El objetivo principal de este estudio es presentar una serie de análisis de los esqueletos de las poblaciones yámana y selk'nam, así como sus medidas antropométricas. Ambos grupos tuvieron distintas formas de cazar, recolectar alimentos y movilizarse en sus territorios. El presente análisis se centra en las proporciones corporales y en el físico de los habitantes originales de Tierra del Fuego, comparándolos con otras poblaciones alrededor del mundo, a fin de estimar de qué manera dichas actividades impactaron su morfología esqueletal.

Cuando los exploradores europeos comenzaron a visitar Tierra del Fuego, encontraron distintos grupos que habitaban la isla, los que incluían a canoeros yámana a lo largo de las costas rocosas del sur y suroeste, kawéskar en el espacio comprendido entre el golfo de Penas y el estrecho de Magallanes, o sea a lo largo de la costa norte, y cazadores terrestres Selk'nam en las planicies norteñas de Tierra del Fuego (Borrero 2001, Bridges 2000). A mediados de 1800, los misioneros ingleses se establecieron en Ushuaia. Desde ese momento los contactos entre europeos y yámana se hicieron más frecuentes, al visitar la misión de Ushuaia distintas expediciones del Viejo Mundo. El contacto con los selk'nam tomó más tiempo debido a que habitaban el interior de la isla, y a que tenían reputación de feroces y aguerridos a diferencia de los más pacíficos yámana (Bridges 2000). Una vez que los estancieros comenzaron a colonizar la zona norte de Tierra del Fuego, la cultura selk'nam declinó rápidamente como resultado de los conflictos directos con dichos estancieros y de la introducción de enfermedades europeas (Borrero y McEwan 1997, Martinic 1997). Los selk'nam se dividían habitualmente en pequeñas bandas, formando grandes grupos cuando existía concentración de recursos, sin embargo, las relaciones entre estos grandes grupos fue por lo general hostil (Gusinde 1982, 1989; Bridges 2000), especialmente una vez que los europeos colonizaron el norte de la isla y empezaron a ejercer una presión considerable sobre los selk'nam del norte (Stuart 1977). Una de las mejores informaciones antropológicas sobre estos grupos proviene de los trabajos de Martín Gusinde quien registró sus datos en las primeras décadas del siglo XX, en el momento en que estas culturas estaban declinando rápidamente por lo que probablemente su comportamiento ya no era igual al que tuvieron antes del contacto con los europeos (Gusinde 1982, 1989; Martinic 1997; Orquera 2002).

Desde los primeros contactos con los yámana y luego con los selk'nam, los observadores europeos se asombraron ante la habilidad de los nativos para vivir en climas fríos y húmedos, usando vestimentas rudimentarias y habitando en viviendas simples. Posteriormente los fisiólogos también se interesaron en este fenómeno, encontrando que los kawéskar mantenían mejor su temperatura corporal que los europeos al permanecer con los brazos sumergidos en agua fría (Hammel 1960). Las poblaciones humanas adaptadas a la vida en climas fríos tienen un físico y unas proporciones corporales diferentes de aquellas poblaciones adaptadas a climas cálidos (Roberts 1978; Trinkaus 1981; Ruff 1994; Pearson 2000). A pesar que Gusinde (1989) y otros registraron una buena cantidad de datos antropométricos para las poblaciones de Tierra del Fuego (Henckel 1950), no se ha estudiado el físico de estas poblaciones con relación a su adaptación al clima. Asimismo, la mayoría de los datos disponibles en la literatura relativa a los habitantes de Tierra del Fuego, consiste en análisis de los esqueletos de los yámana de la costa del sur (Martin 1894; Hyades y Deniker 1891; Garson 1880) 
sin incluir a los selk'nam, con la excepción de Hultkrantz (1907). Hernández et al. (1997) demostraron que algunos aspectos de la morfología craneana, tal como la nariz relativamente alta y proporcionalmente estrecha, probablemente se desarrolló como adaptación al clima frío. Uno de los propósitos de este estudio es determinar el grado de adaptación al clima frío en los esqueletos postcraneales de selk'nam y yámana, en relación con otras poblaciones.

El segundo propósito de este estudio es investigar los efectos de las actividades habituales en el esqueleto humano. Los selk'nam y los yámana nos proveen la oportunidad de comparar dos poblaciones cercanas que vivieron bajo el mismo clima, pero que difieren en sus estrategias de subsistencia y por tanto, en sus actividades físicas. El tercer propósito de este estudio es evaluar el comportamiento de los parámetros vistos en la muestra esqueletal utilizando medidas antropométricas recolectadas por Gusinde a inicios de este siglo.

Las adaptaciones climáticas pueden crear confusión sobre ciertos aspectos de la morfología esqueletal, tales como la robustez de las extremidades, la que se considera generalmente que refleja actividad (Collier 1989; Pearson 2000). El comparar las poblaciones de Tierra del Fuego ofrece el potencial de controlar los efectos del clima sobre las mismas y descubrir las diferencias morfológicas debidas únicamente a los estilos de vida. Las estrategias de subsistencia de selk'nam y yámana han sido documentadas etnográficamente.

El énfasis principal de este estudio está centrado en las poblaciones selk'nam y yámana. La población kawéskar se halla escasamente representada en la muestra esquelética por lo cual no fue considerada en el análisis osteológico. Asimismo, los datos somatométricos son fragmentarios y el uso de la población kawéskar sirve para complementar los datos de la muestra yámana, en tanto que las dos comparten el modo de vida de canoeros.

\section{ESTRATEGIAS DE SUBSISTENCIA DE SELK'NAM Y YÁMANA (Y KAWÉSKAR)}

Los relatos registrados por viajeros tempranos, europeos, misioneros y antropólogos indican que las estrategias de subsistencia de los selk'nam y de los yámana son muy diferentes a pesar de que ambos grupos fueron cazadores-recolectores nómadas. Los selk'nam vivieron en las planicies del norte de la isla en hábitats que se asemejan a las planicies de la Patagonia. Muchas de las bandas también se aventuraban hacia los bosques de los alrededores del lago Fagnano y hacia la sierra Lucio López. Su alimento consistía principalmente en recursos terrestres, aunque los grupos cuyos territorios incluían partes de la costa también recolectaron mariscos. Los hombres selk'nam se especializaban en la caza del guanaco, pero también incluían entre sus presas a zorros, roedores y una variedad de aves (Gusinde 1982, 1989; Stuart 1977; Bridges 2000). Estas actividades requirieron que los hombres realizaran caminatas y rastreos considerables. Su arma primordial fue el arco y la flecha. No usaron trampas con la excepción de lazos utilizados para atrapar ciertas especies de aves. Las bandas de los selk'nam tendían a moverse de manera frecuente.

Los yámana y los kawéskar han sido descritos como cazadores-recolectores marinos, aunque se aventuraban en tierra firme para recolectar algunos vegetales y cazar mamíferos terrestres en los bosques de Nothofagus. Ambos grupos vivieron a lo largo de la línea costera y utilizaron canoas para viajar a lo largo de la costa. La recolección de moluscos era realizada por las mujeres y constituía la mayor parte de la dieta, la que se suplementaba con las aves y mamíferos marinos que cazaban los hombres. Los kawéskar cazaban pocos mamíferos marinos y muchas aves, a diferencia de los yámana los cuales por lo general permanecían en sus campamentos entre 2 a 3 semanas. Tanto hombres como mujeres remaban las canoas durante jornadas migratorias. Sin embargo, al efectuar una estimación diaria, las mujeres yámana remaban más que los hombres además de realizar las actividades características para su subsistencia (Stuart 1972, 1980).

\section{HIPÓTESIS}

Debido al clima frío y húmedo en el que vivieron los habitantes de esta región y a las marcadas diferencias entre los modos de vida yámana ( $\mathrm{y}$ kawéskar) y selk'nam, se puede plantear una serie de hipótesis en relación a cómo sus físicos (y sus esqueletos) deberían reflejar los procesos de adaptación a corto y a largo plazo. La adaptación a largo plazo, es decir al clima frío, ocurre mayormente a lo largo de cientos de generaciones como respuesta al clima de la isla (y quizás también al clima del sur 
de la Patagonia). La adaptación a corto plazo se refiere a las demandas físicas y mecánicas impuestas por las actividades habituales y debió ocurrir en un período de tiempo mucho más corto, a lo largo de la vida de cada individuo.

\section{CLIMA}

Las poblaciones de Tierra del Fuego deberían parecerse a las poblaciones del Viejo Mundo que habitan en zonas climáticas similares - especialmente a las de Europa del este - y no deberían estar tan extremadamente "adaptadas al frío" en términos de sus proporciones corporales si se comparan con poblaciones tales como las inuit de Alaska o las sami del norte de Escandinavia, cuyos ancestros han vivido en el Ártico durante miles de años.

Muchos aspectos del físico se hallan correlacionados con el clima y más posiblemente con adaptaciones a largo plazo al estrés del calor o del frío. Las variaciones en el físico corresponden a las reglas de Bergmann y Allen y actúan para incrementar la proporción del área de la superficie en climas calientes y para disminuir esta proporción en climas fríos (Roberts 1978; Ruff 1994). Las poblaciones de climas fríos tienden a tener una gran masa corporal y extremidades cortas en relación con la estatura (Roberts 1978; Ruff 1994; Holliday 1997), bajos índices crural (largo de la tibia dividido por largo del fémur) y braquial (largo del radio versus largo del húmero) (Trinkaus 1981), y una pelvis ancha en relación con la estatura, tanto de manera absoluta como relativa (Ruff 1994). Los índices del tamaño de los extremos articulares de huesos largos en relación con su largo (índices de robustez epifisial) así como los índices del grosor de los huesos largos en relación con su largo (índices de robustez diafisial) tienden a ser mayores en las poblaciones de áreas frías (Pearson 2000).

Asimismo Ruff (1994) ha propuesto que las proporciones de los huesos de las extremidades, tales como los índices crural y braquial, han evolucionado más rápidamente en respuesta al clima que el ancho pélvico. Esta idea se basa en el hecho de que existe más variación entre los nativos de América en los índices crurales que en la relación bi-iliaca con respecto a la estatura (Ruff 1994). De esta manera los índices crural y braquial son más interesantes para el estudio de la adaptación humana al clima. El índice braquial tiende a ser sexualmente dimórfico pero el índice crural no es sexualmente dimórfico (Trinkaus 1981). El índice crural ofrece la ventaja de que tanto los datos de hombres como de mujeres pueden ser integrados a una misma muestra. En el análisis que se presenta a continuación, sólo se analiza el índice crural debido a esta ventajosa propiedad.

\section{ACTIVIDAD}

Muchos aspectos de la forma y fortaleza de las diáfisis de los huesos largos así como los lugares de inserción muscular responden a las actividades habituales de cada individuo durante su vida. Las formas de las diáfisis de los huesos largos - que incluyen al índice pilástrico de las dimensiones a la altura de mitad de la diáfisis del fémur, el índice cnémico de la diáfisis de la tibia a nivel del agujero nutricio y los índices a la altura de la parte media de las diáfisis del húmero, radio y ulna - puede que respondan a la actividad (Ruff 1987, 2000). Muchos estudios se han centrado en la geometría de los cortes transversales de los huesos de las extremidades (Ruff 2000), sin embargo los índices sobre las dimensiones de las diáfisis generalmente arrojan resultados muy similares a los estudios sobre las fuerzas de torsión de los planos perpendiculares de los huesos (Ruff 1987). La misma lógica subyace a ambos tipos de datos: los huesos crecen de manera tal que acomodan el estrés mecánico que experimentan durante la vida.

Otro importante grupo de índices que puede aportar datos sobre el nivel de actividad habitual son las medidas de "fortaleza residual". Estos índices han sido a veces mal interpretados al llamarlos "robustez", la cual es distinta y se ve principalmente influenciada por la masa corporal en relación con la longitud del hueso (Pearson 2000). La fortaleza residual ha sido medida por lo general a partir de la fuerza de torsión de la diáfisis (segundo momento de área de la sección del módulo de la diáfisis del hueso) de acuerdo con lo establecido por la geometría de cortes transversales. Los segundos momentos de área son luego ajustados, al dividirse por la longitud del hueso y por la masa corporal del individuo para proveer una aproximación sobre de la fuerza del hueso en relación al tamaño del individuo (Ruff 2000). Actualmente, la geometría de cortes transversales provee la mejor manera para estudiar las adaptaciones mecánicas en huesos prehistóricos. 
Debido a que los segundos momentos de área de los cortes transversales están altamente correlacionados $(r>0.90)$ con las dimensiones externas (Pearson 2000), las medidas antero-posterior y medio-lateral de la mitad de la diáfisis (diámetros mayores y mínimos) pueden ser utilizados para representar la fortaleza de la diáfisis con una precisión razonable y a un costo mucho menor que el "escaneo" de tomografía computarizada. Un método sencillo de aproximarse a la fortaleza residual consiste en dividir las dimensiones de la diáfisis del hueso por las dimensiones de las epífisis (Ruff 2000), que es la aproximación que se ha utilizado en este estudio.

Además de medir los huesos largos se efectuó un número limitado de observaciones sobre el grado del desarrollo de las inserciones musculares en los huesos de las extremidades. La cuantificación de las inserciones musculares en los huesos y su interpretación sigue siendo controversial. Las notas sobre inserciones musculares no fueron hechas siguiendo ningún protocolo establecido para evaluar los marcadores de estrés músculo-esqueletal (Hawkey y Merbs 1995). Sin embargo, el grado de desarrollo de las inserciones musculares, modo casi certero, puede revelar algo acerca de las actividades físicas efectuadas durante la vida del individuo, aunque saber exactamente qué es lo que revelan sigue siendo desconocido (Jurmain 1999).

Debido a los diferentes estilos de vida entre selk'nam, yámana (y kawéskar), se generaron varias hipótesis de acuerdo a cómo deberían diferir los esqueletos. Se esperaba que los selk'nam tuvieran piernas fuertes a la vez que brazos menos desarrollados, patrón que se reflejaría en valores altos de fortaleza residual de las extremidades inferiores y valores bajos de la fortaleza residual de las extremidades superiores. Se esperaba que las intensas caminatas que implica la caza terrestre, produjeran altos índices pilástricos y bajos índices cnémicos en los selk'nam (ambos índices reflejan el mayor fortalecimiento antero-posterior de las diáfisis de los huesos largos en vez del medio-lateral (Ruff 1987). Se esperaría que hubiera mayores diferencias entre los índices pilástrico y cnémico de hombres y mujeres selk'nam que entre los yámana, debido a la dependencia de los hombres selk'nam sobre la caza terrestre.

De acuerdo con las descripciones de los relatos etnográficos sobre los grupos canoeros ha sido posible establecer la hipótesis de que ellos tuvieron piernas menos desarrolladas y brazos fuertes. Sus patrones de actividad debieron resultar en una alta fortaleza residual del húmero, tanto en hombres como en mujeres en comparación con la baja fortaleza residual de sus extremidades inferiores. Se puede predecir que los yámana y los kawéskar debieron tener una proporción inferior entre los diámetros mínimos y máximos en la porción media de las diáfisis de las extremidades superiores. Esta premisa también se apoya en el hecho de que el remar durante una cantidad de tiempo importante debe requerir el uso reiterado de la musculatura de la parte superior de los brazos, la cual incluye al músculo deltoides en los húmeros y los flexores de los dedos y muñeca en el antebrazo. Las acciones de estos músculos deben actuar incrementando por una parte el desarrollo de la cresta del deltoides en el húmero (y el diámetro máximo de la diáfisis en su parte media) así como la proyección de la cresta interósea en el radio y la ulna (y por lo tanto los diámetros máximos de estas diáfisis). Debido a que las mujeres yámana tuvieron principalmente a su cargo la responsabilidad de remar (Stuart 1972, 1980), puede esperarse que sus niveles de fortaleza residual del húmero y sus inserciones musculares de las extremidades superiores sean notablemente mayores que aquellas de las mujeres selk'nam. Se espera que los grupos canoeros de ambos sexos tengan índices pilástricos menores en el fémur y mayores índices cnémicos en la tibia (ambos debieran reflejar una disminución debido a las menores caminatas) que los selk'nam, así como una disminución en la diferencia de dichas proporciones entre hombres y mujeres.

\section{MATERIALES Y MÉTODOS}

Para poder poner a prueba estas hipótesis, los esqueletos selk'nam y yámana de Tierra del Fuego fueron medidos en el Instituto de la Patagonia (Punta Arenas), el Museo Etnográfico de Buenos Aires y el Museo del Fin del Mundo (Ushuaia). Además se consideraron las medidas de selk'nam y yámana publicadas por Hultkrantz (1907) y Hyades y Deniker (1891). Las medidas antropométricas de los grupos selk'nam, yámana y kawéskar fueron tomadas de Gusinde (1989). Las muestras esqueléticas y antropométricas se presentan en la Tabla 1 . No se dispone de fechados radiocarbónicos para la muestra 
esqueletal. Sólo algunos de los esqueletos provienen de contextos documentados, otros pertenecen a individuos que fallecieron en el siglo XIX y sólo escasos individuos están asociados con artefactos históricos (p. e. esqueleto \#50103 Bahía Felipe). El esqueleto femenino de Punta Santa Ana fue incluido entre los yámana debido a la similitud de su morfología de tamaño, inserciones musculares y patrones de artritis. En los demás casos hemos supuesto que la antigüedad de los esqueletos no es considerable y que pueden ser asociados con la documentación etnográfica del siglo XIX, sobre los grupos que habitaron las regiones de la isla donde estos restos fueron hallados. El número total de esqueletos medido efectivamente y el número total de individuos que Gusinde pudo medir es pequeño. Sin embargo, resulta significativo que la muestra aquí analizada constituya la mayor muestra de esqueletos selk'nam disponible en la literatura. Las muestras káweskar y yámana se complementan en tanto que los dos grupos son canoeros.

TABLA 1. Muestras esqueléticas y antropométricas.

Medidas Esqueléticas

\begin{tabular}{|c|c|c|c|}
\hline Grupo & Hombres & Mujeres & Anotaciones \\
\hline kawéskar & 1 & 1 & No están incluidos en el análisis. \\
\hline selk'nam & 11 & 3 & 3 individuos de Hultkrantz (1907). \\
\hline yámana & 7 & 7 & $\begin{array}{c}\text { 1 hombre y 1 mujer de Hultkrantz (1907); } \\
\text { 1 hombre y 1 mujer de Hyades y Deniker (1891). }\end{array}$ \\
\hline inciertos & 2 & 2 & No incluidos en el análisis. \\
\hline
\end{tabular}

Medidas antropométricas (Gusinde, 1989)

\begin{tabular}{|c|c|c|c|c|c|c|}
\hline Medida & selk'nam & selk'nam & yámana & yámana & kawéskar & kawéskar \\
\hline & hombres & mujeres & hombres & mujeres & hombres & mujeres \\
\hline Estatura & 24 & 22 & 14 & 19 & 15 & 16 \\
\hline Anchura bi-iliaca & 20 & 9 & 9 & 14 & 14 & 13 \\
\hline Anchura bi-iliaca/estatura & 20 & 9 & 9 & 14 & 14 & 13 \\
\hline Índice del brazo & 15 & 0 & 0 & 7 & 11 & 9 \\
\hline Índice de pierna & 15 & 0 & 0 & 7 & 7 & 9 \\
\hline
\end{tabular}

Información adicional en relación a las medidas antropométricas está disponible en Gusinde (1989). Gusinde utiliza el nombre 'Halakwulup' para referirse a los Kawéskar.

TABLA 2. Medidas Datos Osteológicos

Clavícula: longitud máxima, diámetro superior-inferior a la mitad de la diáfisis, diámetro dorso-ventral a la mitad de la diáfisis

Húmero. longitud máxima, diámetro máximo vertical de la cabeza del húmero, diámetro máximo a la mitad de la diáfisis,

Húmero: diámetro mínimo a la mitad de la diáfisis.

Radio: longitud máxima, longitud articular, diámetro máximo de la cabeza del radio, diámetro máximo a la mitad de la diáfisis, diámetro mínimo a la mitad de la diáfisis.

longitud máxima excluyendo el proceso estiloides, longitud articular (punto más distal de la superficie articular

Cúbito: excluyendo el proceso estiloides hasta el centro de la incisura semilunar), diámetros máximo y mínimo a la mitad de la diáfisis (en base a la longitud articular).

Pelvis: anchura bi-iliaca (medida luego de articular el sacro y los huesos coxales).

Fémur: longitud máxima, longitud bicondilar (articular), diámetro vertical máximo de la cabeza del fémur, diámetros anteroposterior y medio-lateral (en base a la longitud máxima).

longitud (a la mitad de la superficie articular proximal del cóndilo medial al punto más distal del maléolo medial),

Tibia: longitud articular (a la mitad de la superficie articular proximal del cóndilo medial al centro de la superficie articular con el astrágalo), anchura máxima medio-lateral de la epífisis proximal de la tibia, diámetros antero-posterior y mediolateral a la altura del agujero nutricio de la diáfisis.

Datos antropométricos (Gusinde 1989)

\begin{tabular}{|l|}
\hline Estatura \\
\hline Anchura bi-iliaca (ancho de las caderas a lo largo de las crestas iliacas) \\
\hline Índice de la anchura bi-iliaca dividida por la estatura. \\
\hline Índice del brazo: circunferencia dividida por la longitud del brazo. \\
\hline Índice de la pierna: circunferencia de la pantorrilla dividida por la longitud de la pierna. \\
\hline
\end{tabular}

Ver Gusinde (1989) para detalles adicionales 
Los esqueletos de Tierra del Fuego (Tabla 2) fueron comparados con poblaciones procedentes de diversas partes del mundo (Pearson 2000) cuya muestra incluye aproximadamente 25 hombres y 25 mujeres para cada una de las siguientes poblaciones: recolectores khoisan, pastores de Sudáfrica, horticultores zulú, trabajadores de Sudáfrica, afroamericanos, euro-americanos, cazadores-recolectores del mesolítico europeo de Francia y Escandinavia, pastores sami de renos del norte de Noruega, inuit de Alaska, trabajadores chinos de fábricas de envasados del cementerio de Karluk. Por otra parte el Dr. Chris Meiklejohn nos proporcionó generosamente las medidas postcraneales de varios esqueletos mesolíticos del sitio Vedbaek de Dinamarca.

Se utilizaron los análisis T-test y ANOVA (Sokal y Rolf 1995) para comparar los grupos. Los análisis estadísticos fueron realizados en JMP versión 4.04 (SAS Institute 2001). Debido al gran número de com- paraciones que eran posibles de establecer entre las poblaciones, sólo se reporta el significado estadístico de un número selecto de éstas, poniendo mayor énfasis en los patrones generales arrojados por los análisis.

\section{RESULTADOS}

\section{CLIMA}

En la Tabla 5 se presentan los resultados del índice crural. Los datos de los grupos de Tierra del Fuego se presentan a la izquierda del gráfico (Fig. 1). Los datos de las demás poblaciones se presentan a la derecha, organizando los grupos según zonas climáticas que van desde climas cálidos a la izquierda al frío extremo del Ártico a la derecha. Un esquema en forma de diamante rodea el promedio (en el centro del diamante) y la desviación estándar del promedio de cada muestra. Los diamantes

TABLA 3. Anchura bi-iliaca versus longitud de fémur y estatura

\begin{tabular}{|l|c|c|c|c|}
\hline \multicolumn{1}{|c|}{ Grupo } & Sexo & Anchura bi-iliaca & $\begin{array}{c}\text { Longitud máxima del } \\
\text { fémur }\end{array}$ & $\begin{array}{c}\text { Anchura bi-iliaca / } \\
\text { longitud del fémur }\end{array}$ \\
\hline & & Promedio SD (n) & Promedio SD (n) & Promedio SD (n) \\
\hline selk'nam & hombres & $286.519 .3(8)$ & $430.715 .8(7)$ & $66.46 .0(6)$ \\
\hline yámana & hombres & $272.23 .7(5)$ & $427.118 .0(7)$ & $63.42 .9(5)$ \\
\hline inuit & hombres & $282.414 .6(33)$ & $435.127 .5(37)$ & $64.73 .7(33)$ \\
\hline Afr. Amer. & hombres & $255.916 .5(35)$ & $471.026 .5(41)$ & $54.73 .4(35)$ \\
\hline selk'nam & mujeres & $294.0---(1)$ & $426.513 .4(2)$ & $67.4--(1)$ \\
\hline yámana & mujeres & $256.512 .0(2)$ & $405.032 .4(3)$ & $63.410 .0(2)$ \\
\hline
\end{tabular}

Abreviaturas: Afr. Amer., afro-americanos

Todas las dimensiones en milímetros

TABLA 4. Medidas antropométricas

\begin{tabular}{|c|c|c|c|c|c|c|c|c|c|c|}
\hline Grupo & Sexo & \multicolumn{3}{|c|}{ Estatura en vivos } & \multicolumn{3}{|c|}{ Anchura bi-iliaca en vivos } & \multicolumn{3}{|c|}{$\begin{array}{l}\text { Anchura bi-iliaca / } \\
\text { estatura en vivos }\end{array}$} \\
\hline & & \multicolumn{3}{|c|}{ Promedio SD (n) } & \multicolumn{3}{|c|}{ Promedio SD (n) } & \multicolumn{3}{|c|}{ Promedio SD (n) } \\
\hline selk'nam & hombre & 173 & 5.0 & $(24)$ & 33 & 3.3 & $(20)$ & 19.1 & 1.9 & $(20)$ \\
\hline yámana & hombre & 159.7 & 6.9 & $(14)$ & 31.1 & 1.9 & (9) & 19.3 & 1.4 & (9) \\
\hline kawéskar & hombre & 154.7 & 6.6 & $(15)$ & 29.2 & 2.5 & $(14)$ & 19.0 & 2.0 & $(14)$ \\
\hline yámana \& kawéskar & hombre & 157.1 & 7.1 & (29) & 30.0 & 2.4 & (23) & 19.2 & 1.8 & (23) \\
\hline inuit $^{2}$ & hombre & 165.0 & 6.6 & $(37)$ & 30.6 & 1.7 & (33) & 18.5 & 0.9 & (33) \\
\hline Afr. Amer. ${ }^{2}$ & hombre & 169.7 & 5.6 & $(41)$ & 27.5 & 1.9 & $(35)$ & 16.3 & 1.0 & $(35)$ \\
\hline selk'nam & mujer & 160.6 & 3.6 & $(22)$ & 36.8 & 3.29 & (9) & 22.8 & 1.5 & (9) \\
\hline yámana & mujer & 147.9 & 5.1 & $(19)$ & 33.0 & 3 & $(14)$ & 22.6 & 2.0 & $(14)$ \\
\hline kawéskar & mujer & 143.2 & 3.1 & $(16)$ & 27.7 & 2.6 & $(13)$ & 19.4 & 1.8 & (13) \\
\hline yámana \& kawéskar & mujer & 145.7 & 4.9 & (35) & 30.4 & 3.8 & (27) & 21.0 & 2.4 & $(27)$ \\
\hline
\end{tabular}

Datos de Gusinde (1989) excepto los de los afro-americanos y los Inuit.

1 Combinados.

2 La anchura bi-iliaca y estatura estimada siguiendo a Ruff et al. 1997.

Abreviaturas: Afr. Amer., afro-americanos. 
que no se sobreponen corresponden a diferencias estadísticamente significativas entre los promedios (SAS Institute 2001).

La Tabla 3 muestra los resultados de las comparaciones entre la proporción del ancho bi-iliaco dividido por la longitud del fémur. Los datos comparativos de las poblaciones inuit (adaptación al clima frío) y afro-americanos (adaptación al clima cálido) provienen únicamente de individuos masculinos. En todas las poblaciones las mujeres tienden a tener pelvis anchas en relación con su estatura (Ruff 1994), de manera que los datos comparativos son más relevantes en los hombres de Tierra del Fuego. Los resultados indican claramente que tanto los yámana como los selk'nam tienen pelvis extremadamente anchas en relación con la longitud del fémur y resultan similares en este aspecto al físico de los inuit.
La Tabla 4 muestra las medidas antropométricas de Gusinde de anchos bi-iliacos y estatura de las tres poblaciones de Tierra del Fuego y las compara con medidas derivadas de los esqueletos, utilizando el método de Ruff et al. (1997), de las poblaciones inuit y afro-americanos. Las poblaciones de Tierra del Fuego poseen una pelvis extremadamente ancha en relación con su estatura y son similares en este aspecto a la población inuit adaptada al Ártico.

La Tabla 5 presenta los resultados de las comparaciones de los índices de robustez del fémur y de los extremos proximales de húmeros, radios y tibias entre los pobladores de Tierra del Fuego y las demás poblaciones mundiales. Los resultados obtenidos arrojan un patrón consistente. Los grupos de Tierra del Fuego son muy similares unos con otros. Desde una perspectiva a nivel mundial, selk'nam y yámana

TABLA 5. Índices de robustez epifisial y del índice crural

\begin{tabular}{|c|c|c|c|c|c|}
\hline Grupo & Fémur Prox. & Tibia Prox. & Húmero Prox. & Radio Prox. & Índice Crural* \\
\hline & Prom. SD (n) & Prom. SD (n) & Prom. SD (n) & Prom. SD (n) & Prom. SD (n) \\
\hline \multicolumn{6}{|l|}{ HOMBRES } \\
\hline selk'nam & $\begin{array}{lll}10.8 & 0.3(7)\end{array}$ & $\begin{array}{lll}23.2 & 1.7 & (4)\end{array}$ & $14.5 \quad 0.8(8)$ & $\begin{array}{llll}9.6 & 0.4 & (4)\end{array}$ & $80.94 .5(6)$ \\
\hline yámana & $10.8 \quad 0.4(6)$ & $\begin{array}{lll}22.9 & 0.3 & (4)\end{array}$ & $14.4 \quad 0.5(7)$ & $10.5 \quad 0.3(4)$ & $82.4 \quad 1.4$ (4) \\
\hline australianos & $\begin{array}{lll}9.8 & 0.6 & (18)\end{array}$ & $20.3 \quad 1.3(18)$ & $\begin{array}{lll}13.8 & 0.7 & (17)\end{array}$ & $9.4 \quad 0.6$ (17) & $83.3 \quad 2.7 \quad(24)$ \\
\hline khoisan & $\begin{array}{llll}9.7 & 0.4 & (27)\end{array}$ & $20.51 .0(21)$ & $13.10 .8(21)$ & $9.5 \quad 0.6(23)$ & $83.6 \quad 2.3 \quad(35)$ \\
\hline Afr. Amer. & $10.10 .6(41)$ & $20.91 .2(41)$ & $\begin{array}{lll}14.2 & 0.7 & (41)\end{array}$ & $\begin{array}{lll}9.9 & 0.6(41)\end{array}$ & $82.22 .6(72)$ \\
\hline zulú & $10.0 \quad 0.5(31)$ & $20.8 \quad 1.0 \quad(31)$ & $\begin{array}{llll}13.7 & 0.7 & (31)\end{array}$ & $9.50 .5(31)$ & $83.5 \quad 3.0 \quad(62)$ \\
\hline chinos & $19.40 .4(27)$ & $\begin{array}{lll}21.7 & 1.2(25)\end{array}$ & $\begin{array}{lll}14.1 & 0.7 & (25)\end{array}$ & $9.90 .6(26)$ & $80.8 \quad 2.2(26)$ \\
\hline Eur. Amer. & $10.70 .6(25)$ & $22.2 \quad 1.4(25)$ & $\begin{array}{lll}14.9 & 0.8 & (25)\end{array}$ & $\begin{array}{lll}10.4 & 0.7 & (25)\end{array}$ & $80.0 \quad 2.4(50)$ \\
\hline mesolíticos & $11.0 \quad 0.6(8)$ & $23.1 \quad 1.3(3)$ & $\begin{array}{lll}15.3 & 0.9 & (6)\end{array}$ & $\begin{array}{llll}9.9 & 0.8 & (6)\end{array}$ & $81.12 .0 \quad(12$ \\
\hline sami & $11.40 .6(33)$ & $23.9 \quad 1.4(28)$ & $\begin{array}{lll}14.9 & 0.9 & (33)\end{array}$ & $\begin{array}{lll}10.6 & 0.7 & (29)\end{array}$ & $77.32 .2(58)$ \\
\hline inuit & $11.0 \quad 0.5(62)$ & $23.3 \quad 1.2(47)$ & $14.60 .7 \quad(43)$ & $\begin{array}{lll}10.1 & 0.7 & (43)\end{array}$ & $78.62 .3(86)$ \\
\hline \multicolumn{6}{|l|}{ MUJERES } \\
\hline selk'nam & $9.90 .4(2)$ & $21.5 \quad 0.6(2)$ & $13.10 .6(2)$ & $\begin{array}{llll}9.0 & 0.4 & (3)\end{array}$ & ver arriba \\
\hline yámana & $11.0 \quad 0.5(2)$ & $22.3 \quad 1.1 \quad(2)$ & $\begin{array}{lll}14.0 & 0.2 & (3)\end{array}$ & $9.60 .6(2)$ & \\
\hline australianos & $\begin{array}{lll}9.1 & 0.3 & (6)\end{array}$ & $\begin{array}{lll}18.7 & 0.7 & (6) \\
\end{array}$ & $12.5 \quad 0.5(8)$ & $\begin{array}{llll}8.9 & 0.3 & (6)\end{array}$ & \\
\hline khoisan & $9.40 .5(20)$ & $19.81 .1 \quad(17)$ & $12.7 \quad 0.8(20)$ & $\begin{array}{lll}9.2 & 0.7 & (19) \\
\end{array}$ & \\
\hline Afr. Amer. & $9.4 \quad 0.5 \quad(31)$ & $19.81 .1 \quad(31)$ & $13.3 \quad 0.7(31)$ & $\begin{array}{lll}9.1 & 0.7 & (30)\end{array}$ & \\
\hline zulú & $9.5 \quad 0.5(31)$ & 20.11 .1 (31) & $\begin{array}{lll}13.0 & 0.7 & (31)\end{array}$ & $\begin{array}{lll}9.1 & 0.5 & (30)\end{array}$ & \\
\hline Eur. Amer. & $9.90 .6(25)$ & $20.9 \quad 1.4(25)$ & $13.8 \quad 0.6(25)$ & $9.7 \quad 0.6(25)$ & \\
\hline mesolíticos & $10.4 \quad 0.5 \quad(8)$ & $21.1 \quad 1.1 \quad(3)$ & $14.4 \quad 0.6 \quad(4)$ & $\begin{array}{llll}9.3 & 0.6 & (5)\end{array}$ & \\
\hline sami & $\begin{array}{llll}10.7 & 0.4 & (25)\end{array}$ & $\begin{array}{lll}22.9 & 1.2(24) \\
\end{array}$ & $13.90 .8(24)$ & $\begin{array}{lll}9.9 & 0.7 & (23) \\
\end{array}$ & \\
\hline inuit & $10.5 \quad 0.4(25)$ & $22.6 \quad 1.2(24)$ & $13.8 \quad 0.8(25)$ & $9.7 \quad 0.6$ (23) & \\
\hline
\end{tabular}

Abreviaturas: Prox., proximal; Afr. Amer., afro-americanos; Eur. Amer., europeo-americanos.

Fórmula para los índices (todas multiplicadas por 100): Fémur proximal; diámetro vertical de la cabeza del fémur dividido por la longitud máxima del fémur. Tibia proximal: anchura máxima medio-lateral de la epífisis proximal dividida la longitud articular (la distancia desde la mitad del cóndilo medial al centro de la superficie articular distal). Húmero proximal: diámetro vertical de la cabeza del húmero dividido su máxima longitud. Radio proximal: diámetro máximo de la cabeza del radio dividido por la longitud articular (la distancia desde el medio de la superficie articular distal al medio de la superficie articular proximal de la cabeza del radio) (Pearson 2000).

* Sexos combinados. El índice es calculado 100 veces el total de la longitud de la tibia (a la mitad de superficie proximal del cóndilo medial al punto más distal del maléolo medial) dividido por la longitud total del fémur. Este índice difiere del índice crural de Martin y Saller (1957), el cual utiliza la longitud máxima de la tibia. 
poseen huesos largos con extremos articulares grandes en relación con su longitud. De hecho, ambos grupos poseen altos índices de robustez articular que los hace más similares a las poblaciones del Ártico como los inuit de Alaska o los sami del norte de Escandinavia (Pearson 2000). Estas proporciones reflejan la existencia de una gran cantidad de masa corporal en relación con la estatura en todas estas poblaciones. También debe destacarse que en varios casos las muestras de Tierra del Fuego son similares a las de los europeos mesolíticos, las cuales también tienen grandes articulaciones (Fig. 2).

El índice de robustez epifisial del extremo proximal del radio (cabeza del radio) no sigue el patrón general de los huesos largos. Los promedios yámana se encuentran por sobre los selk'nam para el mismo sexo, pero sólo la diferencia entre los hombres posee significancia estadística $(p<0.0092)$. Desde una perspectiva a nivel mundial, los índices de robustez de la cabeza del radio de las muestras de Tierra del Fuego no presentan una tendencia clara y varían desde muy gráciles a muy robustos. Es posible que el pequeño tamaño de la muestra haya sesgado los resultados en ambas muestras de mujeres, pero esto no ocurre con los hombres, los que rodean el promedio (selk'nam) o lo rebasan (yámana) (Tabla 5). Es posible que estas diferencias en el tamaño relativo de las cabezas de los radios de yámana y selk'nam proporcionen un mejor registro osteológico acerca de las diferencias entre sus hábitos de lo que se ha supuesto. Una vez más, la aproximación más cercana a la robustez de la diáfisis de los huesos largos de selk'nam y yámana se halla entre las poblaciones árticas y mesolíticas europeas, las que viven en climas fríos y húmedos (Tabla 6).

\section{ACTIVIDAD}

Las proporciones e índices y otros aspectos de la morfología esqueletal generalmente atribuidas a distintos patrones de actividad arrojaron un patrón mucho menos claro que los resultados debido a las adaptaciones físicas al clima. En la Tabla 5 y la Fig. 3 se presentan los resultados obtenidos al comparar las proporciones de las formas de las epífisis de los

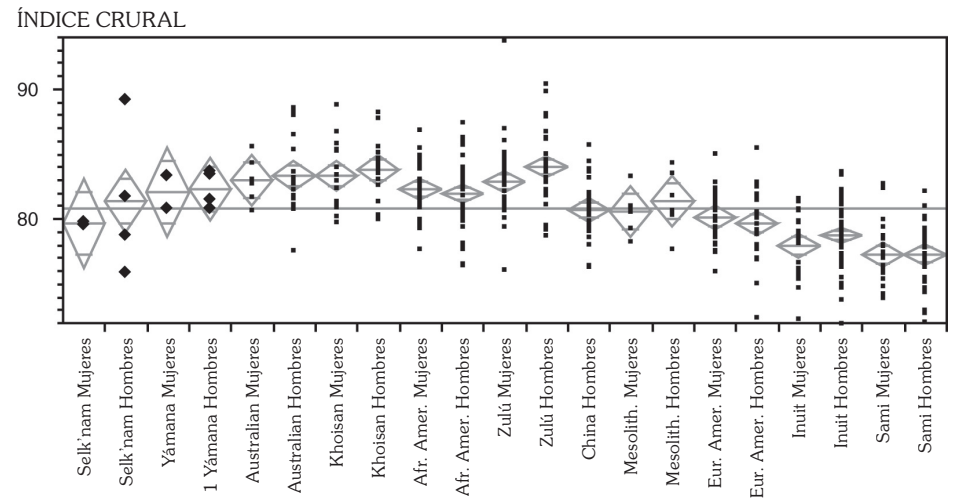

Fig. 1

FÉMUR: ÍNDICE DE ROBUSTICIDAD DE LA EPÍFISIS PROXIMAL

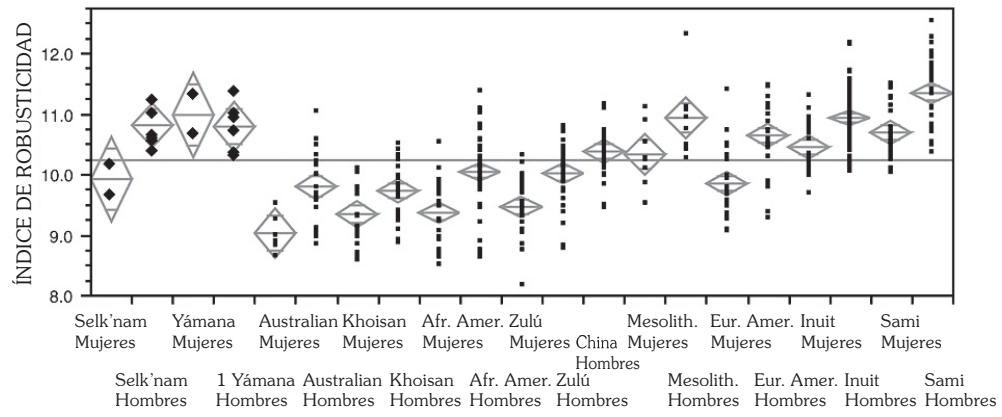

ÍNDICE CNÉMICO

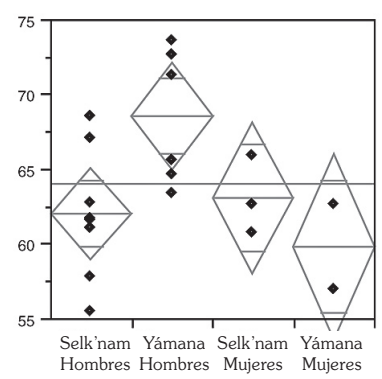

ÍNDICE MITAD DIÁFISIS RADIO

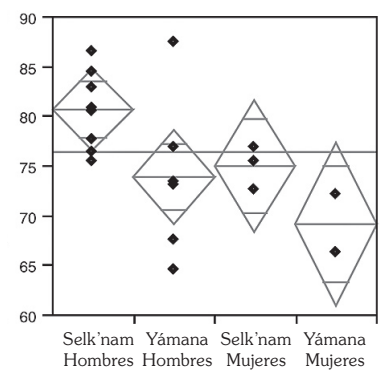

Fig. 3

Fig. 2 
TABLA 6. Índices de robustez diafisial

\begin{tabular}{|c|c|c|c|c|c|}
\hline Grupo & Fem. Rbst. & Tibia Rbst. & Clav. Rbst. & Hum. Rbst. & Radius Rbst. \\
\hline & Prom. SD (n) & Prom. SD (n) & Prom. SD (n) & Prom. SD (n) & Prom. SD (n) \\
\hline \multicolumn{6}{|l|}{ HOMBRES } \\
\hline selk'nam & $13.6 \quad 0.7(7)$ & $16.81 .1(4)$ & $14.40 .3(2)$ & $\begin{array}{lll}13.9 & 1.1 & (8)\end{array}$ & $\begin{array}{lll}12.0 & 1.2 & (8)\end{array}$ \\
\hline yámana & $13.30 .5(6)$ & $16.10 .8(4)$ & $14.70 .3(3)$ & $14.10 .6(6)$ & $12.50 .6(6)$ \\
\hline australianos & $12.20 .7(18)$ & $14.41 .1(18)$ & $17.21 .9(16)$ & 13.20 .8 (17) & $11.51 .0 \quad(17)$ \\
\hline khoisan & $12.50 .7(30)$ & $14.61 .1(23)$ & $14.51 .7(24)$ & $\begin{array}{lll}11.1 & 0.9 & (21) \\
\end{array}$ & $10.31 .0(24)$ \\
\hline Afr. Amer. & $12.60 .8(25)$ & $14.30 .8(41)$ & $16.21 .6(21)$ & $12.8 \quad 0.9(41)$ & $11.9 \quad 1.0(41)$ \\
\hline zulú & $12.50 .6(31)$ & $14.90 .9(31)$ & $15.21 .5(29)$ & $\begin{array}{lll}13.0 & 0.9 & (31)\end{array}$ & $11.41 .0 \quad(31)$ \\
\hline chinos & $12.30 .8(27)$ & $14.60 .8(26)$ & $16.41 .4(26)$ & $12.50 .9(26)$ & $\begin{array}{lll}11.5 & 0.9 & \text { (27) }\end{array}$ \\
\hline Eur. Amer. & $12.60 .8(25)$ & $15.1 \quad 1.2(24)$ & $16.31 .8(25)$ & $\begin{array}{lll}13.1 & 0.9 & (25)\end{array}$ & $\begin{array}{lll}12.6 & 1.1 & (25)\end{array}$ \\
\hline mesolíticos & $13.20 .7(6)$ & $16.20 .7(6)$ & $16.41 .8(4)$ & $\begin{array}{lll}13.8 & 1.3(6) \\
\end{array}$ & $12.31 .2(6)$ \\
\hline sami & $13.0 \quad 0.6(33)$ & $15.50 .9(31)$ & $16.61 .8(30)$ & $\begin{array}{llll}13.7 & 0.9 & (34) \\
\end{array}$ & $13.01 .0 \quad(33)$ \\
\hline inuit & $13.50 .8(47)$ & $16.00 .9(47)$ & $15.01 .7(45$ & $13.5 \quad 1.0(45)$ & $12.2 \quad 1.1$ (45) \\
\hline \multicolumn{6}{|l|}{ MUJERES } \\
\hline selk'nam & $12.90 .7(2)$ & $15.91 .3(2)$ & $12.3---(1)$ & $\begin{array}{lll}11.7 & 0.8 & (2) \\
\end{array}$ & $10.6 \quad 0.9 \quad(3)$ \\
\hline yámana & $13.60 .0(2)$ & $14.70 .0(2)$ & $15.61 .9(2)$ & $12.70 .1 \quad(2)$ & $11.41 .1(2)$ \\
\hline australianos & $11.40 .8(6)$ & $13.60 .7(6)$ & $14.71 .5(7)$ & $10.51 .0(8)$ & $10.0 \quad 0.6(7)$ \\
\hline khoisan & $12.0 \quad 0.7(21)$ & $13.61 .2(19)$ & $13.51 .5(15)$ & $10.8 \quad 0.9(20)$ & $\begin{array}{lll}10.2 & 0.7 & (19)\end{array}$ \\
\hline Afr. Amer. & $12.0 \quad 0.8(31)$ & $13.61 .0(31)$ & $14.61 .2(30)$ & $\begin{array}{lll}12.0 & 1.0 & (31)\end{array}$ & $11.11 .0(31)$ \\
\hline zulú & $12.30 .7(31)$ & $14.31 .0(31)$ & $15.21 .5(29)$ & $\begin{array}{llll}11.9 & 0.9 & (31)\end{array}$ & $\begin{array}{llll}10.8 & 0.7 & (30)\end{array}$ \\
\hline Eur. Amer. & $12.30 .9(25)$ & $13.91 .0(25)$ & $16.31 .8(25)$ & $11.7 \quad 1.2 \quad(25)$ & $11.9 \quad 1.2(25)$ \\
\hline mesolíticos & $12.60 .4(6)$ & $14.10 .9(4)$ & $14.80 .2(4)$ & $12.61 .5(7)$ & $11.30 .9(6)$ \\
\hline sami & $12.60 .8(25)$ & $15.11 .0(24)$ & $15.41 .1(23)$ & $\begin{array}{llll}12.6 & 0.7 & (25)\end{array}$ & $12.8 \quad 1.2(24)$ \\
\hline inuit & $13.0 \quad 0.6(25)$ & $15.0 \quad 0.8(25)$ & $13.61 .3(21)$ & $12.3 \quad 0.8(25)$ & $\begin{array}{lll}11.7 & 0.9 & (25)\end{array}$ \\
\hline
\end{tabular}

Abreviaturas: Rbst., robustez; Fem., fémur; Tib., tibia; Clav., clavícula; Hum., húmero; Afr. Amer., afro- americano; Eur. Amer., Euro-americano Fórmula para los índices (todas multiplicadas por 100); Fémur: diámetro antero-posterior a la mitad de la diáfisis más diámetro medio-lateral a la mitad de la diáfisis dividido la longitud bi-condilar. Tibia: diámetro antero-posterior a la mitad de la diáfisis más diámetro medio-lateral a la mitad de la diáfisis dividido por la longitud articular. Clavícula: diámetro supero-inferior a la mitad de la diáfisis más diámetro dorso-ventral a la mitad de la diáfisis dividido por la longitud máxima. Húmero: diámetro máximo a la mitad de la diáfisis más diámetro mínimo a la mitad de la diáfisis dividido por la longitud máxima. Radio: diámetro máximo a la mitad de la diáfisis más diámetro mínimo a la mitad de la diáfisis dividido por la longitud articular (Pearson 2000).

huesos largos. Opuestamente a lo planteado por nuestra hipótesis, no existen diferencias significativas entre hombres y mujeres selk'nam y yámana en cuanto a sus índices pilástrico, humeral o ulnar. Como se predijo los hombres selk'nam tienen índice cnémico promedio significativamente bajo $(p<0.0173)$ en relación con los hombres yámana, pero los índices de las mujeres yámana no difieren significativamente con respecto a mujeres u hombres selk'nam. Además, el índice de la mitad de la diáfisis radial en hombres yámana, es significativamente menor ( $p>0.0572$ ) que el de los hombres selk'nam, diferencia que corresponde a un mayor desarrollo de la cresta interósea del radio en los hombres yámana. Los promedios de las mujeres yámana resultan nuevamente inferiores a los de los selk'nam, no existiendo diferencias significativas entre las mujeres en el índice radial, resultado que puede atribuirse al pequeño tamaño de la muestra.
La Tabla 7 y la Fig. 4 presentan los resultados de las proporciones de "fortaleza residual" (dimensiones diafisiales divididas por anchos epifisiales). Las definiciones de las medidas incorporadas en estas proporciones se dan más adelante, en la Tabla 7. De modo opuesto a la hipótesis, las proporciones de fortaleza residual femoral de los selk'nam no son significativamente mayores que las de los yámana. Asimismo, tampoco existen diferencias significativas entre hombres o mujeres selk'nam y yámana con respecto a la fortaleza residual de la tibia, sin embargo, la comparación entre mujeres alcanza significancia $(p<0.1529)$, resultado que puede ser producto del reducido tamaño de la muestra de mujeres. En relación con los miembros superiores, no hay diferencias significativas entre hombres 0 mujeres selk'nam y yámana en sus proporciones de fortaleza residual humeral y radial. Finalmente, las proporciones de fortaleza residual del húmero 
TABLA 7. Índices indicativos de patrones de actividad

\begin{tabular}{|c|c|c|c|c|}
\hline & \multicolumn{2}{|c|}{ HOMBRES } & \multicolumn{2}{|c|}{ MUJERES } \\
\hline & selk'nam & yámana & selk'nam & yámana \\
\hline & Prom. SD (n) & Prom. SD (n) & Prom. SD (n) & Prom. SD (n) \\
\hline \multicolumn{5}{|c|}{ FORMAS DIAFISIALES } \\
\hline Pilástrico & $111.7 \quad 11.1 \quad(8)$ & $111.27 .2(6)$ & $101.3 \quad 3.4(2)$ & $101.8 \quad 3.1 \quad(2)$ \\
\hline Cnémico & $\begin{array}{lll}62.1 & 4.3(8)\end{array}$ & $68.64 .5(6)$ & $63.22 .6(2)$ & $59.9 \quad 4.1(2)$ \\
\hline Húmero & $\begin{array}{lll}76.3 & 6.4 & (9) \\
\end{array}$ & 74.13 .4 (6) & $70.8 \quad 1.4 \quad(3)$ & $70.0 \quad 2.2(2)$ \\
\hline Radio & $\begin{array}{lll}80.7 & 3.9 & (8)\end{array}$ & $73.98 .0 \quad(6)$ & $\begin{array}{lll}75.1 & 2.1 & (3)\end{array}$ & $69.3 \quad 4.1(2)$ \\
\hline Cúbito & $74.610 .3(8)$ & $78.09 .0(6)$ & $66.1 \quad 10.4 \quad(3)$ & $68.3 \quad 11.7 \quad(2)$ \\
\hline \multicolumn{5}{|c|}{ ROBUSTEZ RESIDUAL } \\
\hline Fémur & $\begin{array}{lll}12.4 & 0.8(7)\end{array}$ & $12.30 .5(6)$ & $\begin{array}{lll}12.8 & 0.2(2)\end{array}$ & $12.20 .5(2)$ \\
\hline Tibia & $\begin{array}{lll}7.1 & 0.2 & (7)\end{array}$ & $6.90 .4(6)$ & $7.4 \quad 0.4 \quad(2)$ & $\begin{array}{lll}6.6 & 0.3(2)\end{array}$ \\
\hline Húmero & $9.60 .6(8)$ & $9.70 .2(6)$ & $8.9 \quad 0.2(2)$ & $9.0 \quad 0.1 \quad(2)$ \\
\hline Radio & $12.5 \quad 1.0(4)$ & $11.80 .5(4)$ & $\begin{array}{lll}11.7 & 0.6(3) \\
\end{array}$ & $\begin{array}{lll}11.9 & 0.4(2) \\
\end{array}$ \\
\hline Húmero/Fémur & $\begin{array}{lll}7.6 & 0.6(5)\end{array}$ & $\begin{array}{lll}7.9 & 0.2 & (6)\end{array}$ & $6.9---(1)$ & $\begin{array}{lll}7.3 & 0.2(2)\end{array}$ \\
\hline
\end{tabular}

Las fórmulas de los índices de robustez residual (todas multiplicadas por 100); Fémur: diámetro antero-posterior a la mitad de la diáfisis más diámetro medio-lateral a la mitad de la diáfisis dividido por diámetro vertical de la cabeza del fémur. Tibia: diámetro antero-posterior a la mitad de la diáfisis más diámetro medio-lateral a la mitad de la diáfisis dividido por el diámetro máximo medio-lateral de la epífisis proximal. Húmero: diámetro máximo más diámetro mínimo dividido por diámetro supero-inferior de la cabeza del fémur. Radio: diámetro máximo a la mitad de la diáfisis más diámetro mínimo a la mitad de la diáfisis dividido por el diámetro máximo de la cabeza del radio.

FORTALEZA RESIDUAL FÉMUR

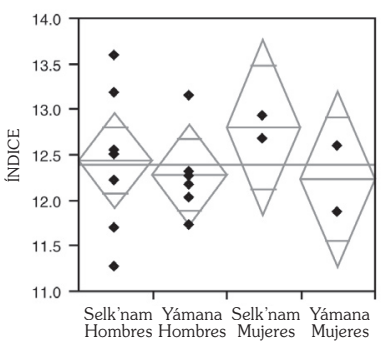

FORTALEZA RESIDUAL HÚMERO

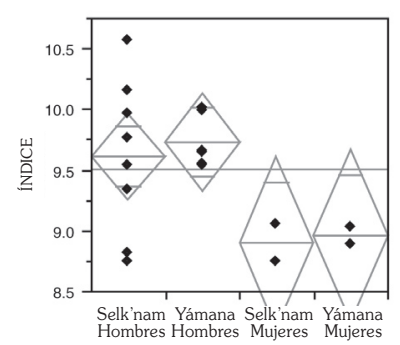

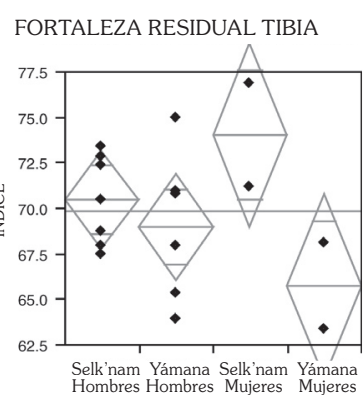

FORTALEZA RESIDUAL RADIO

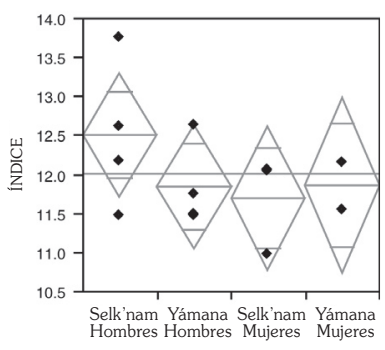

Fig. 4

dividida por la fortaleza residual del fémur resultan de particular interés porque proveen una prueba simple sobre la hipótesis que plantea que los selk'nam debieron tener brazos menos desarrollados y piernas fuertes, mientras que el patrón opuesto caracterizaría a los yámana. Por otra parte, no permite detectar diferencias significativas entre hombres o mujeres, sin

embargo es interesante observar que los promedios se ubican dentro de los patrones esperados, ya que los promedios selk'nam se hallan por debajo de los promedios yámana del mismo sexo. Es posible que la magnitud de las diferencias del estrés mecánico generado por las actividades físicas características de ambos grupos fuera simplemente insuficiente para producir mayores diferencias de fortaleza residual en los huesos de las extremidades.

\section{DATOS ANTROPOMÉTRICOS}

Los datos antropométricos muestran que los selk'nam eran en promedio considerablemente más altos que los yámana. Los índices de las circunferencias de las extremidades por el largo de su segmento proveyeron de una manera simple y estandarizada para evaluar las diferencias en el tamaño del cuerpo. Las diferencias de tamaño del cuerpo entre individuos son comparativamente pequeñas por lo que los cambios alométricos deberían ser de baja magnitud. La medida de las circunferencias de las extremidades es una combinación de las áreas ocupadas por hueso, músculo y grasa siendo que sin la medición de los pliegues cutáneos y los anchos de hombro, rodilla y tobillo (no registrada por Gusinde) resulta muy difícil de estimar en qué magnitud cualquier diferencia en 
el grosor relativo de una extremidad se debe a las diferentes proporciones de los distintos tejidos. Además que puede ser incierto estimar en qué grado las posibles diferencias entre los grupos pueden atribuirse a los cambios surgidos en sus estilos de vida al ingresar a las misiones.

Gusinde (1989) no registró ciertas medidas para hombres yámana, para suplir este déficit hemos incluido los datos de hombres kawéskar a fin de complementar los datos de los canoeros. Las mujeres yámana tienen una diferencia significativa $(p<0.05)$ para el índice del muslo en relación con los hombres selk'nam y con ambos sexos kawéskar. El índice de la pierna de los hombres selk'nam fue mayor (pantorrillas proporcionalmente más gruesas) al de cualquier otro grupo (Fig. 5). Por otra parte, las mujeres yámana tienen pantorrillas más gruesas $(p<0.05)$ que las mujeres kawéskar y son a la vez similares a las de los hombres kawéskar. Se esperaba que el índice del brazo produjera un claro contraste entre los cazadores terrestres y los grupos canoeros. Los datos de Gusinde no arrojan diferencias significativas entre los hombres, sin embargo las mujeres kawéskar y yámana tienen un índice significativamente mayor que los grupos masculinos. Este resultado se puede atribuir en primer lugar a que los brazos de las mujeres yámana son gruesos, con un promedio significativo frente a los kawéskar de ambos sexos. En el índice del antebrazo no se hallaron diferencias significativas entre ambos sexos kawéskar y hombres selk'nam, sin embargo, las mujeres yámana arrojaron un índice significativamente mayor al de cualquier otro grupo. Los datos antropométricos de Gusinde para las cir-

\section{ÍNDICE DE LA PIERNA}

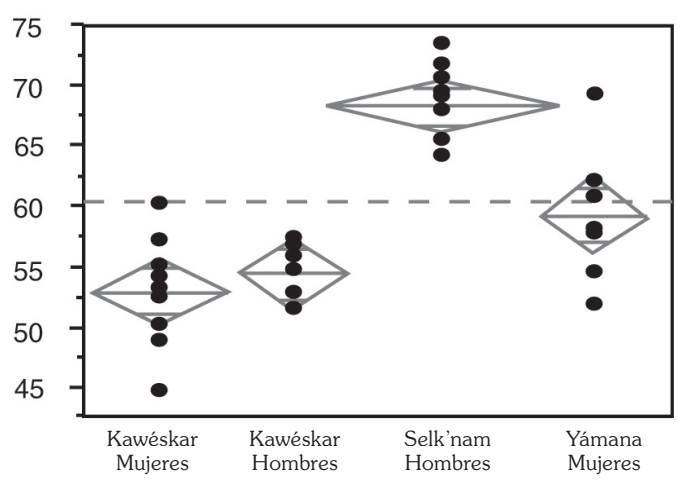

Fig. 5 cunferencias relativas del largo de la pierna entregan contrastes más claros entre selk'nam y yámana (y kawéskar) que los datos obtenidos de la medición de las diáfisis de los huesos largos. Este resultado no será bienvenido por aquellos antropólogos que deseen inferir patrones prehistóricos de actividades pasadas basándose en los esqueletos.

\section{INSERCIONES MUSCULARES}

Los datos sobre el grado de desarrollo de las inserciones musculares no fueron sistemáticamente registrados. Sin embargo, uno de nosotros (OMP) tomó notas sobre el grado de desarrollo de éstas para el pectoral mayor, el deltoides y el redondo mayor en el húmero, así como del grado de desarrollo de la línea áspera y la pilastra en el fémur y de la línea soleal para el origen del sóleo en la tibia. El objetivo de estas notas fue determinar si sería adecuado un estudio más detallado sobre las inserciones musculares. Los resultados muestran que no hay diferencias claras con una excepción importante. Los individuos de ambos grupos tienden a tener un buen desarrollo de la tuberosidad del deltoides $e$ inserciones ásperas para el pectoral mayor. Sin embargo, todos los individuos yámana manifiestan un desarrollo impresionante de la inserción del redondo mayor. La función principal del músculo redondo mayor, es contraer el brazo por lo que su uso es importante al remar, jalar objetos hacia atrás desde la parte anterior del cuerpo y acciones similares. En este caso, las inserciones musculares podrían efectivamente, aportar una imagen clara acerca de las actividades físicas, en contraste con los índices de la forma de la fortaleza residual.

\section{CONCLUSIONES}

En términos de adaptación climática, los selk'nam y yámana ocuparon una posición en el mundo que es comparable con aquellas poblaciones adaptadas al frío. Tanto las medidas del ancho esqueletal bi-iliaco relativo al largo del fémur como las mediciones antropométricas de Gusinde (1989) del ancho bi-iliaco y de la estatura en estas poblaciones vivas, apoyan conjuntamente la conclusión de que tanto selk'nam como yámana tuvieron físicos semejantes a los de las poblaciones adaptadas al Ártico en el hemisferio norte. Tan sólo los índices 
crurales de selk'nam y yámana, relativamente menos extremos, dejan de apoyar la idea de que sus físicos fueran similares a las poblaciones humanas más adaptadas al frío.

Los resultados del análisis de los índices que reflejan actividad arrojan un patrón que resulta menos claro. Se esperaba que las diferencias en los patrones de subsistencia entre selk'nam y yámana hubieran producido marcadas diferencias en la fortaleza de los huesos de sus brazos y piernas y en las formas de los cortes óseos de sus extremidades. Solamente las diferencias en el índice cnémico de la tibia y en la forma de la parte media de la diáfisis del radio se corresponden con estas expectativas. Así, la mayoría de los índices no revelan diferencias entre los grupos. Otro punto importante de este análisis ha sido detectar que los datos antropométricos de Gusinde (1989) muestran diferencias mucho más claras entre selk'nam y yámana que los datos osteológicos. Los índices de la pierna muestran mejor la superioridad de los datos al producir el mismo patrón que el índice cnémico (la esperada diferencia entre selk'nam y yámana), pero de una manera mucho más pronunciada. El hecho que este tipo de contrastes pueda observarse claramente en tejidos blandos, pero que su registro óseo sea mucho menos evidente, es quizás un resultado desalentador para los antropólogos. Finalmente, las observaciones sobre el grado de desarrollo de la inserción del músculo redondo mayor en el húmero indicaría una diferencia mayor y persistente entre selk'nam y yámana. En los yámana adultos de ambos sexos estas inserciones tienen la forma de una cresta ancha y protuberante, mientras que los selk'nam muestran un desarrollo mínimo de esta inserción para ambos sexos. Las diferencias entre ambos grupos son atribuibles a la actividad de remar en canoa, aunque resulta sorprendente que una actividad que deja una huella tan clara en esta inserción muscular no se manifieste de manera más evidente en las diáfisis de los huesos así como en otras variadas inserciones musculares de la extremidad superior.

Las conclusiones de este análisis esqueletal son sorprendentes debido a que muchos investigadores han expresado con gran confianza que estas mediciones pueden revelar detalles confiables acerca de las actividades de los pueblos prehistóricos (Larsen 1995; Ruff 2000). Sin embargo, Jurmain (1999) ha retado el enfoque ampliamente sostenido de que los huesos largos y las formas de sus secciones aportan una imagen clara de las actividades prehistóricas. La crítica de Jurmain se basa en el hecho de que existen muy pocos estudios empíricos que hayan mostrado en qué cantidad una actividad, o bien qué tipo de actividad, produce qué tipo de incremento en la fortaleza de los huesos o qué tipo de cambios en la forma de las epífisis de los huesos. De la misma manera, Bridges (1997) no encontró correlación significativa entre el grado de desarrollo de las inserciones musculares y la estimación de fortaleza del húmero en una muestra de esqueletos prehistóricos de Illinois. La mayoría de los antropólogos hubiera pensado que éstos se encuentran cercanamente correlacionados. Los resultados de Tierra del Fuego acerca de las medidas que reflejan supuestamente patrones de actividad se suman a las críticas de Jurmain y Bridges, demostrando que es necesaria más investigación para aclarar en qué grado los patrones de actividad producen realmente los tipos de modificaciones en la morfología esqueletal que los antropólogos han asumido generalmente como ciertos.

\section{AGRADECIMIENTOS}

Se agradece a los Drs. J. L. Lanata, R. A. Guichón, J. A. Pérez Gollán, I. Baffi, M. I. Gelari, A. Prieto, y a Srs. P. Cárdenas Soto y J. Corbalán y también al Museo Etnográfico de Buenos Aires, al Instituto de la Patagonia y al Museo del Fin del Mundo que conservan las colecciones de restos óseos que formaron la base de este estudio. Agradecemos a los Drs. D. Stuart, Y. M. Lam, T. VanPool, y C. VanPool por proveer comentarios útiles. Los fondos para la investigación de las colecciones de restos óseos humanos alrededor del mundo fueron provistos por la National Science Foundation Fellowship, y por becas de la Wenner-Gren Foundation, Boise Fund, y la University of New Mexico.

\section{BIBLIOGRAFÍA}

BORRERO, L. A. y C. MCEWAN. 1997. The peopling of Patagonia: The first human occupation. En Patagonia: Natural History, Prehistory and Ethnography at the Uttermost End of the Earth. Editado por C. McEwan, L. A. Borrero, y A. Prieto, pp. 32-45. Princeton: Princeton University Press.

BORRERO, L. A. 2001. Los Selk'nam (Onas). Buenos Aires: Editorial Galerna-Búsqueda de Ayllu. 
BRIDGES, E. L. 2000. El Último Confín de la Tierra (translation by María Magdalena Briano of the 1948 original, The Uttermost Part of the Earth). Editorial Sudamericana Buenos Aires.

BRIDGES, P. S. 1997. The relationship between muscle markings and diaphyseal strength in prehistoric remains from West-Central Illinois. American Journal of Physical Anthropology Supplement 24:82.

COLLIER, S. 1989. The influence of economic behaviour and environment upon robusticity of the post-cranial skeleton: a comparison of Australian Aborigines and other populations. Archaeology in Oceania 24:17-30.

GARSON, J. G. 1880. On the inhabitants of Tierra del Fuego. Journal of the Royal Anthropological Society 15:141-160.

GUSINDE, M. 1982, 1989. Los Indios de Tierra del Fuego. Resultado de mis cuatro expediciones en los años 1918 hasta 1924, organizadas bajo los auspicios del Ministerio de Instrucción Pública de Chile. Editorial. Buenos Aires: Centro Argentino de Etnología Americana. Consejo Nacional de Investigaciones Científicas y Técnicas. [Translation of the (1931-1939) German originals, especially (1939) Die Feuerland-Indianer. Ergebnisse meiner vier forschungreisenin den Jahren 1918 bis 1924, unternomen in auftrage des Ministerio de Instrucción Pública de Chile. Band 3. Anthropologie der Feuerland-Indianer. Wien-Mödling Verlag Der Internationalen Zeitschrift Anthropos].

HAMMEL, H. T. 1960. Response to cold by the Alacaluf indians: A first report on a 1959 expedition. Current Anthropology 1:146.

HAWKEY, D. E., y C. F. MERBS. 1995. Activity-induced musculoskeletal stress markers (MSM) and subsistence strategy changes among ancient Hudson Bay Eskimos. International Journal of Osteoarchaeology 5:324-338.

HENCKEL, C. 1950. The anthropometry of the Indians of Chile. En: Handbook of South American Indians, Vol. 6. Editado por J. H. Steward, pp. 121-135. Washington, D. C.: Smithsonian Institution.

HERNÁNDEZ, M., C. LALUEZA FOX y C. GARCÍA-MORO. 1997. Fueguian cranial morphology: The adaptation to a cold, harsh environment. American Journal of Physical Anthropology 103:103-117.

HOLLIDAY, T. W. 1997. Body proportions in Late Pleistocene Europe and modern human origins. Journal of Human Evolution 32:423-447.

HULTKRANTZ, J. V. 1907. Zur Osteologie der Ona- und YahganIndianer des Feuerlandes, in Wissenschaftliche Ergebnisse der schwedischen Expedition nach den Magellensländern 1895-97 unter Leitung von Dr. Otto Nordenskjöld, vol. 1, Geologie, Geographie und Anthropologie, No. 5, pp. 109-173. Stockholm: Norstedt \& Söner.

HYADES, P. y J. DENIKER. 1891. Anthropologie et Ethnographie. En: Mission Scientifique du Cap Horn (1882-1883), Vol. 7. Paris: Gauthier-Villars et Fils.

JURMAIN, R. 1999. Stories from the Skeleton: Behavioral Reconstruction in Human Osteology. Gordon and Breach Publisher, Amsterdam.

LALUEZA F., C., M. HERNÁNDEZ, y C. GARCÍA-MORO. 1996. Craniometric analysis in groups from Tierra del Fuego/Patagonia and the peopling of the south extreme of the Americas. Human Evolution 11:217-224.
LARSEN, C. S. 1995. Biological changes in human populations with agriculture. Annual Review of Anthropology 24:185-213.

MARTIN, R. 1894. Zur physischen Anthropologie der Feuerläender. Archiv für Anthropologie 22:155-218.

MARTIN, R. y K. SALLER. 1957. Lehrbuch der Anthropologie. Stuttgart: Gustav Fischer Verlag.

MARTINIC B., M. 1997. The meeting of two cultures: Indians and colonists in the Magellan region. En Patagonia: Natural History, Prehistory and Ethnography at the Uttermost End of the Earth. Editado por C. McEwan, L. A. Borrero, y A. Prieto, pp. 110-126. Princeton University Press, Princeton.

ORQUERA, L. A. 2002. The late-Nineteenth Century crisis in the survival of the Magellan-Fueguian littoral natives. En Archaeological and Anthropological Perspectives on the Native Peoples of Pampa, Patagonia, and Tierra del Fuego to the Nineteenth Century. Editado por C. Briones y J. L. Lanata, pp. 145-158. Bergin \& Garvey Westport, Connecticut.

PEARSON, O. M. 1997. Postcranial Morphology and the Origin of Modern Humans. Ph.D. thesis, State University of New York at Stony Brook.

PEARSON, O. M. 2000. Activity, climate, and postcranial robusticity: Implications for modern human origins and scenarios of adaptive change. Current Anthropology 41:569-607.

ROBERTS, D. F. 1978. Climate and Human Variability. 2nd edition. Menlo Park, Cummings Massachussets.

RUFF, C. 1987. Sexual dimorphism in human lower limb bone structure: relationship to subsistence strategy and sexual division of labor. Journal of Human Evolution 16:391-416.

RUFF, C. B. 1994. Morphological adaptation to climate in modern and fossil hominids. Yearbook of Physical Anthropology 37:65-107.

RUFF, C. B. 2000. Biomechanical analyses of archaeological human skeletons. En: Biological Anthropology of the Human Skeleton. Editado por M. A. Katzenberg y S. R. Saunders, pp. 71-102. New York: Wiley-Liss.

RUFF, C. B., E. TRINKAUS, y T. W. HOLLIDAY. 1997. Body mass and encephalization in Pleistocene Homo. Nature 387:173-176

SAS INSTITUTE. 2001. JMP®, VERSION 4.04. Cary, North Carolina: SAS Institute, Inc.

SOKAL, R. R. y F. J. ROLF. 1995. Biometry, Third edition. W. H. Freeman \& Company, New York.

STUART, D. E. 1972. Band Structure and Ecological Variability: The Ona and Yaghan of Tierra del Fuego. Ph.D dissertation, University of New Mexico.

STUART, D. E. 1977. Seasonal phases of Ona subsistence, territorial distribution and organization: Implications for the archaeological record. En: For Theory Building in Archaeology. Editado por L. R. Binford, pp. 251-283. Academic Press, New York.

STUART, D. E. 1980. Kinship and social organization in Tierra del Fuego: evolutionary consequences. En: Versatility of Kinship, pp. 269-284. Academic Press, New York.

TRINKAUS, E. 1981. Neandertal limb proportions and cold adaptation. En Aspects of Human Evolution. Editado por C. B. Stringer, pp. 187-224. Taylor \& Francis, London. 